\title{
RELATIVSYNTAGMEN IM DEUTSCHEN UND IN EUROPÄISCHEN VERGLEICHSSPRACHEN: FUNKTIONALE DOMÄNE UND AUSGEWÄHLTE VARIANZPARAMETER
}

\begin{abstract}
Der Beitrag verfolgt zwei Zielsetzungen: eine deskriptive und eine methodologische. Auf der Ebene grammatischer Beschreibung erfolgt eine Analyse der deutschen Relativsatzkonstruktion aus der Gegenüberstellung mit entsprechenden Konstruktionen anderer europäischer Sprachen heraus, insbesondere mit Konstruktionen des Englischen, Französischen, Polnischen und Ungarischen, den Kernkontrastsprachen des Projekts „Grammatik des Deutschen im europäischen Vergleich“. Dabei wird auf die zentralen Projektkonzepte 'funktionale Domäne' und 'Varianzparameter' rekurriert. Die funktionale Domäne des Relativsatzes wird als Beitrag zu der übergreifenden Funktion nominaler Konstruktionen, nämlich der Referenz, bestimmt und zwar als referentielle Modifikation des begrifflichen Kerns durch einen verankernden Sachverhalt. Von den die Sprachen differenzierenden Parametrisierungen werden drei herausgegriffen und in ihrer Korrelation diskutiert.

In methodologischer Hinsicht soll am Beispiel des Relativsatzes gezeigt werden, in welcher Weise typologische Generalisierungen, Kontraste zwischen - in diesem Fall überwiegend nah verwandten bzw. über Sprachkontakte miteinander verbundenen - Sprachen und einzelsprachenspezifische Eigenschaften aufeinander zu beziehen sind, immer im Dienst einer besseren Einsicht in das Funktionieren des Deutschen.

This article has two objectives, one descriptive and the other methodological. On the level of grammatical description, an analysis of the German relative clause construction is undertaken from the point of view of a contrast with corresponding constructions in other European languages, in particular English, French, Polish and Hungarian, the core contrast languages of the project "German grammar in a European comparison". Reference is made to the central concepts of the project, 'functional domain' and 'variance parameter'. The functional domain of the relative clause is defined as a contribution to the general function of nominal constructions, i.e. reference, specifically as referential modification of the conceptual core by the circumstances in which it is embedded. Three parameters which differentiate between the languages are picked out and discussed in their correlation with one another.
\end{abstract}

As far as the methodology is concerned, the example of the relative clause is used to show how typological generalisations, contrasts between languages - in this case predominantly closely related languages or languages which have long been in contact with one another - and characteristics specific to individual languages are connected, always with a view to obtaining a better insight into the way the German language functions.

\section{Worum es geht}

Relativsätze oder, allgemeiner gesprochen, 'Relativsyntagmen' sind gut erforscht, sowohl im Rahmen der Grammatikforschung zum Deutschen als auch aus sprachvergleichender und sprachtypologischer Perspektive. Sie sind daher als Gegenstand der Reflexion über Methoden und Theoriebildung einer typologisch vergleichend fundierten Grammatikschreibung des Deutschen besonders gut geeignet. Nicht von ungefähr waren Relativsätze daher das Thema der Pilotstudie Zifonun (2001), in der das Programm des Projekts „Grammatik des Deutschen im europäischen Vergleich“"1 zum ersten Mal an einem grammatischen Phänomenbereich erprobt wurde.

\footnotetext{
${ }^{1} \mathrm{Zu}$ dem Projekt vgl. auch http://www.ids-mannheim.de/gra/eurostudien.html
} 
Bereits in dieser Studie wurde in Anlehnung an Zifonun (2001a) von den Konzepten der funktionalen Domäne und des Varianzparameters als forschungsleitenden Kategorien ausgegangen. An diesen Konzepten orientiert sich die Projektarbeit nach wie vor. Die Diskussion in der Projektgruppe und die Arbeit an weiteren Phänomenbereichen aus der Grammatik des Nominals hat uns aber ein gutes Stück weiter gebracht. Die eher programmatischen und wenig differenzierten Aussagen über das Konzept der 'funktionalen Domäne' aus diesen Anfängen können wir nun präzisieren, mit vergleichbaren Konzepten verknüpfen und mit empirischem Gehalt füllen, vgl. dazu auch Gunkel/Zifonun (demn).

Eines der Ziele dieses Beitrags ist es, ein Stück weit - nämlich so weit wie für Relativsätze einschlägig und notwendig - über den jetzigen Stand unseres Konzeptes der funktionalen Domäne Rechenschaft abzulegen. Die einzelsprachliche Varianz in der Realisierung einer funktionalen Domäne soll der Projektkonzeption nach über die Kategorie 'Varianzparameter' erfasst werden. So wurden in der Pilotstudie zum Relativsatz insgesamt acht Parameter genannt, die den Spielraum formaler und funktionaler Ausdifferenzierung abstecken. Dabei war uns von Anfang an klar, dass die Variation nicht arbiträr erfolgt, sondern prinzipiengeleitet und zwar vor allem in der Weise, dass die einzelnen Parameterbelegungen aufeinander bezogen, miteinander korreliert sein können. Dieser Gesichtspunkt ist allerdings zunächst nicht, auch in der genannten Studie nicht, deutlich zum Ausdruck gekommen. So konnte der Eindruck entstehen, die Parametersetzung erschöpfe sich, in der Konzeption des Projekts, im deskriptiv-klassifikatorischen Nebeneinander von Einzelfakten. Das zweite Ziel des Beitrags ist es daher, anhand einiger weniger Varianzparameter für den Relativsatz, die wir herausgreifen, systematische Abhängigkeiten, Korrelationen aufzudecken. $\mathrm{Zu}$ wünschen ist, dass die Verfolgung beider methodisch-theoretischer Ziele der eigentlichen Zielsetzung unseres Projekts, nämlich Neues über die Grammatik des Deutschen, hier insbesondere die Grammatik von Relativsätzen, zu erfahren, eher förderlich als hinderlich ist.

\section{Funktionale Domäne}

Für den Sprachvergleich brauchen wir Vergleichsgrößen, an denen wir die spezifischen ausdrucksseitigen, z.B. morphologischen oder syntaktischen Mittel, Marker, Kategorien und Konstruktionen erst messen können. Das Tertium comparationis des Sprachvergleichs ist also, so das Postulat maßgeblicher Typologen (vgl. stellvertretend Croft 2003, S. 13 ff.), auf einer ersten und grundlegenden Ebene ein semantisches oder allgemeiner ein funktionales. Solche sprachübergreifenden pragmatischen oder semantischen Funktionen, auf die Phänomenbereiche natürlicher Sprachen zu beziehen sind, bezeichnen wir mit dem Terminus 'funktionale Domäne' und knüpfen damit an die Begrifflichkeit von Givón (1981), Frajzyngier (1999) sowie Lehmann (2004) an. Allerdings verfügen wir beim derzeitigen Stand der Forschung nicht über eine sprachtheoretisch hinreichend abgesicherte Gesamtsystematik 'funktionaler Domänen', an die wir anschließen könnten. Es ist zu hoffen, dass der Fortschritt typologischer Untersuchungen in einem dialektischen Wechsel von Hypothesenbildung und empirischer Evaluation zunehmend Klarheit darüber verschaffen wird, welche funktionalen Domänen für die natürlichsprachliche Kommunikation grundlegend sind und für die mutmaßlich alle Sprachen grammatikalisierte Realisierungsmöglichkeiten bereitstellen und welche anderen zwar grundsätzlich 
ausdrückbar sind im Sinne des Searleschen Sagbarkeitsprinzips ${ }^{2}$, für die aber ggf. in bestimmten Sprachen oder Sprachgruppen keine eigenen Formen und Muster ausgebildet wurden. Im Hinblick auf die grundlegenden funktionalen Domänen orientieren sich Typologen an den sprachpragmatischen Grundfunktionen von Referenz und Prädikation im Sinne der Searleschen Sprechakttheorie, die als konstitutive Bestandteile propositionaler Akte den Gehalt illokutionärer Akte bestimmen.

Die funktionale Domäne der Referenz, genauer der Referenz auf Gegenstände eines kognitiv erfassten Realitätsausschnittes, ist nun eben die Funktion, die den nominalen Konstituenten, genauer den Nominalphrasen mit substantivischem Kern, unserer Vergleichssprachen als prototypische zugeordnet ist. Damit ist weder behauptet, dass Referenz grundsätzlich nur durch Nominalphrasen geleistet werden kann, noch ist ausgeschlossen, dass Nominalphrasen andere Funktionen, etwa die der Prädikation in Prädikativkonstruktionen, übernehmen können.

Die konstruktionelle Komplexität von Nominalphrasen spiegelt, so unsere Hypothese, ihre funktionale Komplexität wider. Der zentrale Bautyp des Nominals sieht in unseren Vergleichssprachen vor, dass die Kernkonstituente von einem appellativischen Substantiv gebildet wird, um das Attribute unterschiedlicher Ausdrucksklassen (adjektivisches Attribut, Genitivattribut/Possessorattribut, adpositionales Attribut usw.) gruppiert sind und das fakultativ oder obligatorisch durch einen Artikel oder ein anderes Determinativ spezifiziert wird wie in folgendem Beispiel jeweils im Deutschen und den vier Kernkontrastsprachen des Projekts, nämlich Englisch, Französisch, Polnisch und Ungarisch:

$$
\begin{aligned}
& \text { DEU das } \text { Det } \text { interessante }_{\text {Attr }} \text { Buch }_{\text {Kern }}[\text { über Europa }]_{\text {Attr }} \\
& \text { ENG the } \text { Det } \text { interesting }_{\text {Attr }} \text { book }_{\text {Kern }}[\text { on Europe }]_{\text {Attr }} \\
& \text { FRZ le } \text { Det }_{\text {Divre }} \text { Kern }_{\text {intéressant }} \text { Attr }_{\text {[sur l'Europe }]_{\text {Attr }}} \\
& \text { POL interesująca } a_{\text {Attr }} k_{\text {ssiążka }} a_{\text {Kern }}[o \text { Europie }]_{\text {Attr }} \\
& \text { UNG az }{ }_{\text {Det }}[\text { Európáról szóló }]_{\text {Attr }} \text { érdekes } \text { Attr }_{\text {könyv }} \text { Kern }
\end{aligned}
$$

Die drei syntaktischen Grundfunktionen Kern, Attribut und Determinator - in den Beispielen jeweils durch die Indizes 'Kern', 'Attr' und 'Det' markiert - zeigen drei funktionale Subdomänen der Gegenstandsreferenz an: Nomination, Modifikation und Determination (vgl. dazu auch Seiler 1985, 2000; Wiese 2004). Der substantivische appellativische Kern leistet eine erste begriffliche Einordnung: Das Kernsubstantiv nennt einen Begriff - von daher 'Nomination' -, mit dem der Gegenstand, auf den referiert wird, nach Meinung des Sprechers zutreffend charakterisiert wird, wobei normalerweise derjenige Begriff aus einer Vielzahl von möglichen gewählt wird, der im Hinblick auf den zu vollziehenden Referenzakt und den Sprechakt, in die dieser eingebettet ist, am zweckmäßigsten erscheint, das heißt z.B. die Menge der Referenten, die überhaupt im Frage kommen, am effektivsten für den Hörer einschränkt. Das Kernsubstantiv leistet somit aufgrund seiner deskriptiven Bedeutung eine semantische Beschränkung des Referenzpotentials. Die Aufgabe der Attribute besteht darin, durch Bildung komplexer Begriffe, durch 'Modifikation', das Referenzpotential semantisch weiter einzugrenzen. 'Determination' durch Determinatoren wie Demonstrativa oder Artikel hingegen leistet

\footnotetext{
${ }^{2}$ Vgl. Searle (1969), S. 19 f. ,,principle of expressibility“: ,whatever can be meant can be said““.
} 
eine pragmatische Beschränkung des Referenzpotentials, indem etwa durch ein Demonstrativum signalisiert wird, dass der Referent im deiktischen Raum lokalisierbar ist, oder durch den definiten Artikel, dass er für den Sprecher in der gegebenen Situation eindeutig identifizierbar ist.

Bevor wir nun genauer in die Domäne der 'Modifikation' einsteigen, der auch der Relativsatz zuzuordnen ist, sollen zwei Punkte hervorgehoben werden: Es kann der Eindruck entstanden sein, dass eine eineindeutige Abbildung, eine Isomorphie, zwischen syntaktischen Funktionen und funktionalen Subdomänen gegeben sein muss. Dies ist jedoch nicht der Fall. Denken wir z.B. an das adnominale Possessivum. Im Deutschen steht es wie der Artikel am linken Rand der Nominalphrase und ersetzt ihn, kann also nicht mit ihm kookkurrieren; es regiert wie der indefinite Artikel die gemischte Deklination des Adjektivs. Es ist also syntaktisch ein Determinator. Im Italienischen und anderen romanischen Sprachen dagegen verhalten sich die Possessiva wie Adjektive, sie können mit dem definiten und auch dem indefiniten Artikel kookkurrieren und folgen den Artikelwörtern nach; sie sind somit syntaktisch Attribute. Was die semantische Funktion angeht, so leistet das Possessivum im Deutschen Determination, im Italienischen eher nicht. Wohl aber haben die Possessiva in beiden Sprachen einen Gehalt, der in der NP Begriffsmodifikation bewirkt: durch mein in mein Buch wird der Begriff 'Buch' in Beziehung gesetzt zum Sprecher, es erfolgt referentielle Modifikation (vgl. den nächsten Abschnitt). Im Deutschen werden somit, in diesem Fall zwei Funktionen (relativ zu den beiden Domänen Determination und Modifikation) kumulativ ausgedrückt durch einen Ausdruck. Der zweite Punkt, der hervorgehoben werden soll, ist die Tatsache, dass die Determination zu den funktionalen Subdomänen gehört, die in unseren Vergleichssprachen nicht durchgängig grammatikalisiert sind. Während im Deutschen, Englischen, Französischen bei individuativen Appellativa im Singular grundsätzlich ein Artikel als Standardausdruck für Determination gesetzt werden muss, kennt das Polnische wie andere slawische Sprachen keine Artikel (vgl. (1)). ${ }^{3}$

\section{1 $\mathrm{Zu}$ welcher funktionalen Domäne gehört der Relativsatz?}

Relativsätze - wir beschränken uns hier auf die adnominalen Erscheinungsformen, klammern also satz- oder prädikatsbezogene Formen sowie den 'freien Relativsatz' aus - sind modifikativ. Ähnlich wie Holler und Blühdorn (in diesem Band) betrachten wir auch die appositive Variante adnominaler Relativsätze als „strukturell integriert“. Sie fungieren syntaktisch als Attribute, was sie ebenso wie die restriktiven Ausprägungen semantisch zu Modifikatoren qualifiziert. Bedeutet die Einordnung als Modifikatoren, dass Relativsätze funktional dasselbe leisten wie Adjektive, Adjektivphrasen oder Partizipialphrasen? Dies wäre eine Hypothese, die aufgrund der Herleitung von Adjektivphrasen aus relativsatzartigen Konstruktionen in der frühen generativen Theorie nahe liegen könnte (vgl. z.B. Motsch 1966). In der typologischen Literatur werden aber mehrere Arten von Modifikation unterschieden mit jeweils partiell differierenden typischerweise zugeordneten Ausdrucksklassen. Dabei bewegen wir uns sowohl mit Adjektiv/Partizipialphrasen als auch mit Relativsätzen im Bereich dessen, was wir als 'Modifika-

\footnotetext{
3 Im Ungarischen ist nur der definite Artikel voll grammatikalisiert. Die Setzung des indefiniten Artikels kann unter bestimmten Bedingungen, insbesondere in der Position unmittelbar vor dem Finitum unterbleiben.
} 
tion im engeren Sinne' im Gegensatz zum Typ der 'Argumentanbindung' bezeichnen wollen. Während Modifikation im engeren Sinne wie in (das) neue grüne Fahrrad oder (das) Fahrrad, das ich gestern gekauft habe den mit der Nomination bereit gestellten Begriff weiter anreichert, wird bei der Argumentanbindung wie in (der) Vater von Hans, (der) Aufstieg des römischen Reiches, (die) Hoffnung auf Frieden durch die Sättigung des zuvor ergänzungsbedürftigen Ausdrucks überhaupt erst ein Begriff gebildet, der 'referenztauglich' erscheint. Innerhalb des so abgesteckten Bereichs der Modifikation im engeren Sinne unterscheiden wir folgende drei Subtypen:

- qualitative Modifikation: der Modifikator drückt eine Qualität aus,

- quantitative Modifikation: der Modifikator drückt eine Quantität aus,

- referentielle Modifikation: der Modifikator ist referentiell.

Im Folgenden wird uns in erster Linie das Verhältnis von qualitativer und referentieller Modifikation interessieren. Die 'quantitative' Modifikation, im nominalen Bereich in erster Linie durch Numeralia geleistet, soll daher nicht weiter beachtet werden.

Bei qualitativer Modifikation wie etwa in grünes Fahrrad, Frau von dreißig Jahren wird das Gegenstandskonzept um ein Eigenschaftskonzept angereichert. Adjektive sind die prototypischen lexikalischen Realisierungsformen qualitativer Modifikation. Zum Kernbereich der Adjektive gehören sprachübergreifend Ausdrücke für die folgenden Qualitätsdomänen: Dimension ('groß', 'klein'), Alter ('jung', 'alt', 'neu'), Bewertung ('gut', 'schlecht') und Farbe ('weiß', 'rot'). Adjektive dieser Gruppen sind nach Dixon (1977) auch in den Sprachen vertreten, die nur eine kleine, geschlossene Klasse von Adjektiven besitzen. Solche prototypischen Adjektive stellen in erster Line eine begriffliche Erfassung von „relativ einfachen perzeptuellen Eigenschaften, die Individuen besitzen“" (vgl. Lyons 1983, S. 76) dar. Sie sind 'stativ', zeigen also keine Veränderung in der Zeit an, und vergleichsweise 'persistent' (oder auch 'permanent', vgl. Abschnitt 3.1), d.h. sie gelten von einem Gegenstand in der Regel für ein längeres Zeitintervall, vgl. Croft (1991, S. 64 f.). In Sprachen wie u.a. unseren Vergleichssprachen, in denen die Adjektive eine offene und durch produktive Wortbildungsmuster erweiterbare Wortklasse bilden, wird dieser enge Bereich der Zuschreibung einfacher, perzeptueller, stativer und persistenter Qualitäten weit überschritten. Ein interessantes auch in der Semantikforschung viel beachtetes Thema ist dabei die Beobachtung, dass bestimmte Adjektive systematisch schwanken zwischen einer Lesart als Zuschreibung einer persistenten oder inhärenten Eigenschaft und der Charakterisierung eines temporären Zustands, man vergleiche im folgenden Beleg die Vorkommen von normale, nette, witzige mit dem Vorkommen von traurig.

(2) Der Clown, der Blues und das Requiem von Mozart nach Feierabend - manchmal sind auch normale, nette, witzige Kerle traurig wie im Klischee. (Der Spiegel, 06.12.1993, S. 231)

In der Semantik wird dieses Thema durch die Gegenüberstellung von ,individual level“ und ,stage level“"-Prädikationen behandelt (vgl. Kratzer 1989). Auch im Hinblick auf die anstehende Behandlung des Relativsatzes ist zu fragen, wie weit bei der qualitativen Modifikation durch Adjektive vom Prototyp stativer und persistenter Qualitäten in Richtung auf die Zuschreibung auch nicht-stativer (i.e.) dynamischer oder temporärer Eigen- 
schaften abgewichen werden kann. Hier ist insbesondere an die Partizipien zu denken, die in den Vergleichssprachen als ,,adjektivierte“ Verbformen zu begreifen sind.

Nur am Rande sei darauf hingewiesen, dass wie in dem bereits erwähnten Frau von dreißig Jahren oder Mann der Tat, Herz aus Gold usw. auch attributive Phrasen, Nominalphrasen im Genitiv oder Präpositionalphrasen, im Deutschen qualitativ modifizierend sein können. Gerade beim attributiven Genitiv - man spricht bei den entsprechenden Fällen auch vom Genitivus qualitatis oder dem Eigenschaftsgenitiv (vgl. Eisenberg 2004b, S. 249) - handelt es sich aber nicht um eine zentrale Funktion, was unter anderem daran deutlich wird, dass nur eine bestimmte syntaktische Konfiguration, nämlich 'definiter Artikel + Substantiv im Singular' und nur bestimmte mehr oder weniger usuelle Verbindungen auf einem gehobenen Stilniveau überhaupt in Gebrauch sind (vgl. *Mann einer Tat, ?? Mann schlechter Manieren, ${ }^{?}$ Mann weniger Worte ${ }^{4}$ ). Genitivphrasen in der zentralen Funktion als Possessorphrasen wie in (das) Haus meines Vaters, (die) Puppen dieses Kindes oder als Argumente relationaler Substantive wie (der) Vater dieses Kindes, (die) Zerstörung Roms sind als 'referentielle Modifikatoren' einzuschätzen.

Bei referentieller Modifikation wird das Gegenstandskonzept in Relation zu einem Referenten gesetzt. Der Modifikator hat selbstständige Referenzfunktion und der entsprechende Gegenstand befindet sich, so wird präsupponiert, in einer bestimmten Beziehung, z.B. einer Zugehörigkeits- oder Possessorrelation, zu Elementen der Klasse der Gegenstände, für die das Gegenstandskonzept steht. Der Effekt der Modifikation besteht hier in einer Einschränkung der Gegenstandsklasse auf genau die Gegenstände, die zum Referenten in der Possessorrelation stehen. Die referentielle Modifikation wird aus kognitivpragmatischer Sicht in der Literatur (vgl. Taylor 1996) als besonders effektives Verfahren für die Identifizierung des Referenten der Gesamtphrase betrachtet: Weiß ich erst einmal, bei welchem Gegenstand des universe of discourse ich ansetzen muss, z.B. eben bei der Person, die ich als 'dieses Kind' identifizieren kann, so ist der Schritt zur Identifikation von Gegenständen die unter das Konzept 'Puppen der identifizierten Person' fallen, ein gutes Stück geebnet. Koptjevskaja-Tamm (2002), (2003) spricht daher hier von „referentieller Verankerung“ und hebt dabei hervor, dass eine bereits zugängliche bzw. identifizierbare Entität als 'referentieller Anker' für die weitere Interpretation fungiert; bei Rijkhoff (2004, S. 177 ff.) wird weiter verallgemeinernd von „lokalisierenden Modifikatoren“" gesprochen. ${ }^{5}$ Während Possessorphrasen in der Regel Personen als referentielle Anker erfordern, bedient sich die im engeren Sinne lokalisierende Verankerung auch anderer Entitätssorten wie Sachen oder Orte, man vergleiche Mann neben mir, Buch auf dem Tisch, Haus in Frankfurt. Auch Relativsätze wie in den folgenden Beispielen aus unseren Vergleichssprachen fungieren, so unsere Kernhypothese, als referentielle Modifikatoren:

\footnotetext{
${ }^{4}$ Mann weniger Worte ist belegt in Rhein-Neckarzeitung, 02.04.2007.

${ }^{5} \mathrm{Zu}$ beachten ist, dass referentielle Verankerung hier nicht im informationsstrukturellen Sinne ('given' versus 'new') zu verstehen ist. Letzteres ist gemeint, wenn Prince (1981, S. 236) bestimmt: „Brand-new entities themselves seem to be of two types: Anchored and Unanchored. A discourse entity is Anchored if the NP representing it is linked, by means of another NP, or "Anchor", properly contained in it, to some other discourse entity." In ähnlichem Sinne ist auch das „grounding“ in Fox/Thompson (1990) zu verstehen. Bei dem hier vorliegenden Konzept geht es nicht um Diskursreferenten, sondern um Referenzobjekte ,in der Welt“, auf die im Searleschen Sinn Bezug genommen und über die prädiziert wird. Zwischen beiden Vorstellungen gibt es Berührungen, sie sind jedoch klar voneinander zu trennen.
} 
(3) DEU Ich kenne den Mann, der gerade hier arbeitet.

ENG I know the man who is just working here.

FRZ Je connais l'homme qui est en train de travailler ici.

POL Znam mężczyznę, który właśnie tu pracuje.

UNG Ismerem azt a férfit, aki éppen itt dolgozik.

Was ist hier der referentielle Anker? Relativsätze oder auch allgemeiner satzförmige referentielle Modifikatoren können nur über diejenigen Sorten von Entitäten verankern, die mit der semantischen Form von Sätzen kompatibel sind: Relativsätze denotieren Propositionen, deren Wahrheit bei der Anwendung des komplexen Begriffs, bestehend aus Kernsubstantiv und Relativsatz, auf einen Gegenstand präsupponiert - so bei restriktiven Relativsätzen - oder assertiert wird - so bei appositiven. ${ }^{6}$ Mit ihnen wird gesagt, dass aus Sicht des Sprechers als Kondition für das Zutreffen des komplexen Begriffs ein Sachverhalt der Fall ist; sie entwerfen einen Sachverhalt und setzen dessen Faktizität. Die Funktion von Relativsätzen besteht darin, dass über den Referenten, der unter das Gegenstandskonzept fällt, gleichzeitig eine Beteiligung an dem oder eine kontextuelle Bezogenheit auf diesen Sachverhalt prädiziert wird.

Genau diese Idee steckt hinter der Rekonstruktion, die Lehmann (1984) für die semantische Form der Verknüpfung zwischen Kernsubstantiv und Relativsatz postuliert. Die Verknüpfung läuft notwendig über eine ,semantische Leerstelle“, die die Position des Referenten in der Struktur des Sachverhalts markiert. Relativpronomina, wie der in dem folgenden Beispiel sind die syntaktischen Repräsentanten der semantischen Leerstelle. Anhand von Beispiel (3) kann dies so veranschaulicht werden.

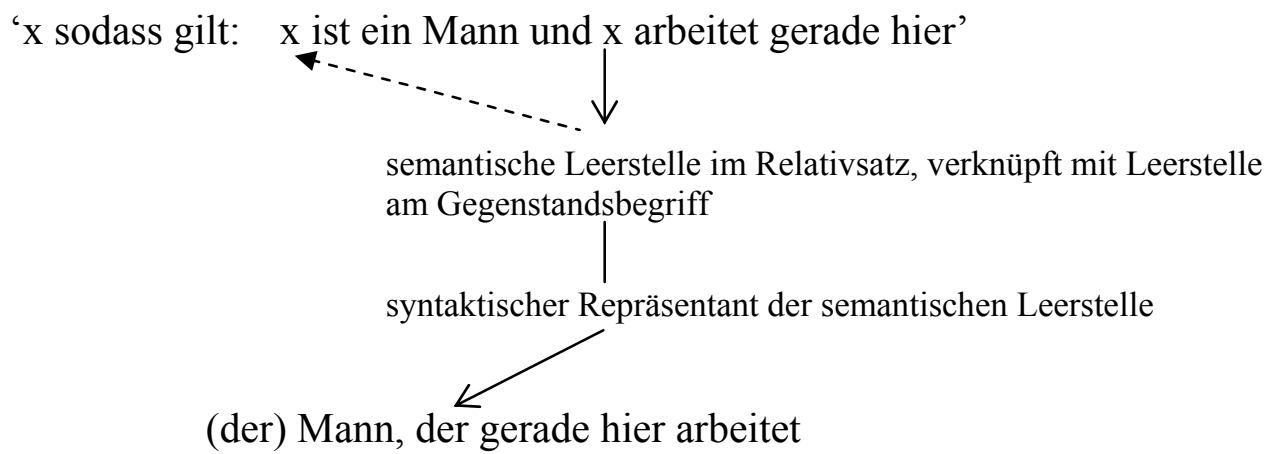

Es ergibt sich somit, dass wir den vom Relativsatz denotierten Sachverhalt als den Faktor betrachten, der zur Identifikation des Referenten der Gesamtphrase beiträgt. Inwiefern kann es nun gerechtfertigt sein, hier von 'referentieller Verankerung' bzw. referentieller Modifikation zu sprechen? Wie bereits hervorgehoben, ist mit Relativsätzen immer ein Wahrheitsanspruch für den Sachverhalt/die Proposition als Voraussetzung für die Anwendung des komplexen Begriffs verbunden. Der Sachverhalt ist also selbst als

\footnotetext{
${ }^{6}$ Bei Eigennamen und deiktischen Personalpronomina, an die appositive Relativsätze angeschlossen werden, ist es strittig, ob hier überhaupt von 'Gegenstandsbegriffen' zu sprechen ist. Wir schließen uns der Auffassung an, es handle sich hier um Grenzfälle, bei denen das entsprechende Individuenkonzept zwar keinen (oder kaum einen) deskriptiven Gehalt habe, dennoch sei von einer konzeptuellen Bedeutung auszugehen, es handle sich um 'Individuenkonzepte mit direkter Referentialität' (Lerner/Zimmermann 1991, S. 355). Vgl. zu dieser Problematik auch den Beitrag von Blühdorn (in diesem Band).
} 
verankert in der Welt, die der Sprecher als wirklich annimmt, zu betrachten; er kann seinerseits ein an ihm beteiligtes Individuum verankern.

$\mathrm{Zu}$ beachten ist hier, dass ein in diesem Sinne verankernder Sachverhalt nicht nur dann vorliegt, wenn die Gesamtphrase spezifisch referentiell ist, wie in (4a), sondern auch, wenn nicht-spezifischer (4b) oder prädikativer Gebrauch (4c) vorliegt:

(4a) Ein Besucher des Straßenfestes in Mannheim-Neckarau, der den Unfall beim Festzelt beobachtet hat, hat sich bei der Polizei gemeldet.

(4b) Besucher des Straßenfestes in Mannheim-Neckarau, die den Unfall beim Festzelt beobachtet haben, sollen sich bei der Polizei melden.

(4c) Herr Maier ist ein Besucher des Straßenfestes in Mannheim-Neckarau, der den Unfall beim Festzelt beobachtet hat. Er hat sich bei der Polizei gemeldet.

Bei spezifischer Verwendung wie in (4a) wie auch bei prädikativer Verwendung (4c) ist schlicht vorausgesetzt, dass der vom Relativsatz denotierte Sachverhalt zutrifft.

Bei nicht-spezifischer Verwendung wie in (4b) gibt es möglicherweise gar keine Personen, die unter den komplexen Begriff fallen. Personen, die wir als „,meldepflichtig“ identifizieren, müssten aber in jedem Fall unter den Begriff 'Besucher des Straßenfestes in Mannheim-Neckarau' fallen und sie müssten den Unfall beim Festzelt beobachtet haben. Der Sachverhalt 'Besucher X hat Unfall beobachtet' muss auf sie zutreffen - wenn es sie denn gibt. Es reicht nicht aus, dass allein der 'Gedanke' im Fregeschen Sinne, der nicht als wahr bewertete Sachverhalt, ins Spiel gebracht wird.

In der Literatur wird statt von Propositionen/Sachverhalten von „Situationen“ oder Ereignissen gesprochen. Man vergleiche dazu Lehmann (1984, S. 402), wo es heißt:

„Mit einem Relativsatz kann man leicht einen bestimmten Gegenstand durch Spezifikation der Situation, an der er teilhat, identifizieren.“

Rijkhoff (2004, S. 175) spricht pauschal von Ereignissen:

„(...) relative clauses designate events, which are located in time, and since the referent of a relativized $\mathrm{NP}$ is necessarily a participant of the event denoted by a relative clause, this participant is located in time (and space) as well.“

Rijkhoff hat damit nur „Sachverhalte in der Zeit“, die Lyons (1983, S. 71) als Entitäten zweiter Ordnung einstuft, als den Relativsätzen korrespondierende referentielle Anker im Auge. Auf Ereignisse bezieht man sich mit Verbalsätzen, die unterschiedliche Zeitstruktur von Ereignissen und die unterschiedlichen Konstellationen der Ereignisbeteiligung spiegeln sich partiell in der Wahl unterschiedlicher Verbtypen wider, wenn wir von Handlungen/Handlungsverben, Vorgängen/Vorgangsverben oder Zuständen/Zustandsverben sprechen. Ereignisse der ,äußeren Welt" sind sicherlich die naheliegendsten und möglicherweise für die Identifikation effektivsten Anker. Doch ,Sachverhalte in der Zeit und der uns umgebenden Welt" sind keineswegs die einzigen Sachverhalte, die durch Relativsätze beschrieben werden. Relativsätze beziehen sich auch auf „innere Zustände“ (5), auf Sachverhalte in einer von der wirklichen Welt her für den Sprecher zugänglichen „möglichen Welt“ des Erwünschten, Gesollten usw. (6) und präsupponieren bzw. assertieren vor allem auch das Nicht-Zutreffen von Sachverhalten (7): 
(5) der Mann, der immer nur an seine Tochter dachte

(6) der Mann, der das Fürchten lernen sollte

(7) der Mann, der die Konferenz nun doch nicht eröffnet

Das Nicht-Eröffnen einer Konferenz ist kein Ereignis. Unter anderem wird das daran erkennbar, dass eine Nominalisierung, die eher in der Form Nicht-Eröffnen als in der Form Nicht-Eröffnung erfolgt, nicht als Subjekt-Argument von Ereignis-Prädikaten wie stattfinden, geschehen auftreten oder durch spatiotemporale Angaben modifiziert werden kann:

(8) *Das Nicht-Eröffnen der Konferenz durch Kanzlerin Merkel findet morgen in Frankfurt statt/dauert voraussichtlich eine halbe Stunde.

gegenüber:

(8a) Die Eröffnung der Konferenz durch Kanzlerin Merkel findet morgen in Frankfurt statt/ dauert voraussichtlich eine halbe Stunde. ${ }^{7}$

Im Hinblick auf Relativsätze werden wir also allgemeiner von einer Verankerung in als wahr vorausgesetzten oder assertierten Sachverhalten sprechen müssen und dabei offen lassen, ob die verankernden Sachverhalte als Ereignisse (in der realen oder einer möglichen Welt) betrachtet werden können.

Daneben aber haben wir zwei Dinge in Rechnung zu stellen:

- Relativsätze können in allen engeren Vergleichssprachen als Prädikatsausdruck auch ein prädikatives Adjektiv (oder auch ein substantivisches Prädikatsnomen) enthalten. ${ }^{8}$ Dieses kann wie in seiner nicht-sententialen attributiven Verwendung sowohl eine Zustands- als auch eine im engeren Sinne qualitative Lesart haben, etwa wenn generell gültige Charakteristika oder Dispositionen ausgedrückt werden wie in folgendem Beleg:

(9) Inzwischen hatte sich der Hirsch mit seinem Geweih, das viel zu imposant war, zwischen den Bäumen verhangen, er lief nicht weg, stand, ein einzigartiger Verstoß gegen das Naturgesetz. (Kleine Zeitung, 28.04.1998)

- Relativsätze konkurrieren mit Partizipialphrasen, bei denen Vollverben - die den finiten Prädikatsausdruck des Relativsatzes bilden würden - in die adjektivierte Form des Partizips konvertiert und zusammen mit ihren Komplementen und Supplementen dem Kernnomen vergleichbar einer Adjektivphrase attribuiert werden, wie in:

(10) Was den von Autofahrern gefürchteten Marder nachts in die Motorräume treibt, hat Forstamtsleiter Klaus Velbecker im Gespräch mit dem „Südhessen Morgen“ erklärt. (Mannheimer Morgen, 01.11.2000)

\footnotetext{
${ }^{7}$ Ironisierend sind solche Verwendungen möglich: Dann wird etwas, das nicht passiert ist, zum Ereignis umgedeutet.

${ }^{8}$ Daraus folgt natürlich, dass nur diejenigen Adjektive in Relativsätze „umgewandelt“ werden können, die selbst prädikativ gebraucht werden können. Zu den Adjektiven ohne prädikativen Gebrauch gehören im Deutschen „nicht-intersektive“ wie z.B. angeblich, mutmaßlich; aber auch abgeleitete Adjektive wie chemisch, medizinisch in medizinische Behandlung, chemische Reaktion können nicht bedeutungserhaltend in einen Satz mit prädikativem Adjektiv umgewandelt werden.
} 
In vielen Sprachen sind nicht-finite Formen, bei denen das Prädikatsverb notwendig in partizipialer oder allgemeiner nominalisierter Form auftritt, die einzigen Formen in denen referentielle Modifikation mittels Verankerung in einem Sachverhalt ausgedrückt werden kann. In der typologischen Literatur werden auch diese Formen zu Recht dem Relativbereich zugeordnet, ich spreche daher allgemeiner von Relativsyntagma, das finit (als 'Relativsatz') oder infinit (als 'Relativinfinit') realisiert sein kann. Wir gehen auf diesen Varianzparameter im nächsten Abschnitt ein.

Der erstgenannte Punkt, Relativsätze mit Adjektiv und Kopulaverb, deutet auf die bereits erwähnte Tatsache hin, dass funktionale Domänen und Ausdrucksklassen praktisch niemals in einer Eins-zu-eins-Korrelation aufeinander bezogen sind (vgl. Rijkhoff 2006). Offenbar können Relativsätze auch Adjektiven vergleichbar qualitative Modifikation ausdrücken. Umgekehrt müssen wir annehmen, dass durch Partizipialphrasen, also Modifikatoren mit ggf. adjektivischer Morphosyntax, referentielle Modifikation ausgedrückt wird. ${ }^{9}$ Es kann also nur darum gehen, die jeweils zentralen oder prototypischen Funktionen, die ggf. das Nebeneinander von Ausdrucksklassen erklären helfen, von den peripheren, die uns auch Überlappungen zwischen den Ausdrucksklassen zeigen, zu unterscheiden. Auf dieses in ein Mehr und Weniger abgestufte Verhältnis im Hinblick auf die Funktionen von Adjektiv und Relativsatz weist schon Lehmann (1984, S. 405) hin: „Das Adjektiv dient mehr der Begriffsbildung, der Relativsatz mehr der Gegenstandsidentifikation.“

\section{Varianzparameter}

Von den acht Varianzparametern, die in Zifonun (2001) behandelt werden, sollen hier zum einen der Parameter 'Relativsatz oder Relativinfinit' aufgegriffen werden, zum anderen die drei Parameter 'Subordination', 'Leerstellenfüllung' und 'Hierarchie syntaktischer Funktionen' in ihrer wechselseitigen Abhängigkeit.

\subsection{Relativsatz oder Relativinfinit}

Alle unsere engeren Vergleichssprachen verfügen über Relativsyntagmen mit finiten Verben, also Relativsätze. In folgenden Sprach(grupp)en sind dagegen ausschließlich oder dominant Relativinfinite vertreten: drawidische Sprachen (z.B. Tamil), Quechua (eine Sprachgruppe der südamerikanischen Anden) sowie einer Reihe von uralischen und altaischen Sprachen. Im europäischen Raum ist das Türkische (als altaische Sprache) der wichtigste Vertreter.

Im Türkischen erfolgt Satzeinbettung generell bevorzugt in nominalisierter Form. Attributive Relativsyntagmen werden pränominal gestellt und haben zwei Ausprägungen, die von der syntaktischen Funktion des syntaktischen Repräsentanten der semantischen Leerstelle im Relativsyntagma determiniert sind. Handelt es sich um die Subjektsfunktion (11) oder um ein (Possessor-)Attribut zum Subjekt (12), so wird das Partizipialsuffix (PPS) - An ('A' für den jeweils unter Vokalharmonie passenden Vokal) gewählt, handelt es sich um eine andere syntaktische Funktion, steht das Nominalisierungssuffix (NGS) -DIK (als Repräsentation allomorphischer Varianten), vgl. (13):

\footnotetext{
9 Die von Eigennamen und Verwandtschaftsbezeichnungen abgeleiteten 'Possessivadjektive' des Russischen wie in mámina kómnata 'Mutters Stube' sind ebenfalls referentiell.
} 
(11) [okul-a gid-en] çocuk

Schul-Dat geh-PPS Kind

'das Kind, das zur Schule geht' wörtlich: 'das zur Schule gehende Kind'

(12) [oğl-u okul-agid-en] adam

Sohn-3Sg Schul-Dat geh-PPS Mann

'der Mann, dessen Sohn zur Schule geht'

(13) $[$ Ahmed-in

git-tiğ-i] okul

Ahmed-Gen geh-NGS-3Sg Schule

'die Schule, in die Ahmed geht' wörtlich: 'die Schule von Ahmeds Gehen' (vgl. Kornfilt 1990, S. 250)

Partizipial- und Nominalisierungssuffix sind somit im Türkischen nach Maßgabe syntaktischer Funktionen im Wesentlichen komplementär verteilt. Das Partizipialsuffix deckt den Subjektbereich, und damit die hierarchiehöchste syntaktische Funktion (vgl. den nächsten Abschnitt) ab, das Nominalisierungssuffix die übrigen syntaktischen Funktionen. Mit dieser Beschränkung auf den Subjektbereich ist auch sprachübergreifend die syntaktische Reichweite von adnominalen Partizipialkonstruktionen im Vergleich zu Relativsyntagmen umrissen: Sie attribuieren grundsätzlich im Subjektbereich, so im Deutschen über das Aktivsubjekt wie beim Partizip Präsens (14) oder beim Partizip Perfekt „unakkusativischer“ und reflexiver Verben (15) oder über das Passivsubjekt wie beim Partizip Perfekt transitiver Verben (16):

(14) ein aus dem Stall fliehendes Pferd $\approx$ ein Pferd, das aus dem Stall flieht

(15) ein in die Jahre gekommener Junggeselle $\approx$ ein Junggeselle, der in die Jahre gekommen ist / ein morgens frisch gewaschener Mensch $\approx$ ein Mensch, der sich morgens frisch gewaschen hat

(16) ein vom Autor schlecht redigierter Text $\approx$ ein Text, der vom Autor schlecht redigiert worden ist

Alle anderen syntaktischen Funktionen des syntaktischen Repräsentanten der semantischen Leerstelle werden in Sprachen, die überhaupt über Relativsätze verfügen und die bezüglich dieser syntaktischen Funktionen relativieren können (vgl. den nächsten Abschnitt), mit eben diesen satzförmigen Konstruktionen ausgedrückt, Partizipialkonstruktionen sind dort nicht möglich: ${ }^{10}$

(17) ein Unternehmer, dem alles gelingt $\approx$ *ein alles gelingender Unternehmer

Partizipien sind Formen zwischen Verb und Adjektiv. Morphologisch werden sie in adnominaler Verwendung in den Vergleichssprachen teilweise analog zu Adjektiven behandelt; teilweise aber zeigen sich Abweichungen: Sie weisen im Polnischen und Deutschen Kasus-, Genus- und Numeruskongruenz mit dem Kernsubstantiv auf, im Englischen und Ungarischen bleiben sie wie die Adjektive unflektiert. Das Französische unterscheidet beim Partizip Präsens eine vollständig adjektivierte kongruierende Form (les collines environnantes 'die umgebenden Hügel') von einer unflektierten Form bei Kom-

\footnotetext{
${ }^{10}$ Im Prinzip sind Partizipialkonstruktionen auch zu anderen Komplementen möglich. Voraussetzung ist, dass das Komplement durch eine Diathese zum Subjekt-Argument promovierbar ist. Allerdings ist diese Voraussetzung nicht immer hinreichend, wie das deutsche bekommen-Passiv zeigt. Beispiele wie

?? ein das Spielzeug weggenommen bekommendes Kind sind kaum akzeptabel und im Korpus nicht belegt.
} 
plementanbindung (les collines environnant la ville 'die die Stadt umgebenden Hügel'). Generell kann sprachabhängig in der Morphosyntax das verbale Erbe mehr oder weniger deutlich durchschlagen. ${ }^{11}$ Halten wir zunächst fest, dass Partizipien funktional - mit der Beschränkung auf die Attribuierung über das Subjekt - eher den Relativsyntagmen als den prototypischen Adjektiven entsprechen: Sie drücken typischerweise eine referentielle Modifikation mittels Verankerung in einem Sachverhalt aus, nicht qualitative Modifikation. Dies gilt besonders dann, wenn durch Komplemente und Supplemente/Adjunkte erweiterte Partizipialphrasen vorliegen, die eine komplexe Sachverhaltskonfiguration erfassen können. Damit korreliert im Deutschen, dass Partizipialkonstruktionen mit Adjektiv und Partizip des Kopulaverbs sein hochgradig markiert sind. Die im Korpus belegten Formen sind meist dadurch motiviert, dass ein nicht flektierbares Adjektiv (oder ein anderer unflektierbarer Modifikator wie z.B. super) auf diese Weise ,analytisch flektiert" wird, vgl.

(18) (...) was, soweit lässt sich der Text systematisieren, etwas mit dem dort vertretenen Ideal des debilen, aber happy seienden Bürgers $\mathrm{zu}$ tun habe (...). (die tageszeitung, 21.07.2001)

Im Englischen hingegen sind Kombinationen von being + Adjektiv in dieser Funktion eher gebräuchlich; wir kommen auf die Unterschiede noch zurück.

(19) That was great and started a whole year of being in America for me, which was seeing David as a major star and also for myself, experiencing life as it should be as a major star, with your cars and people looking after you and the record company being polite to you rather than treating you like shit and not working. (BNC/AB5 „In other words") ${ }^{12}$

Betrachtet man die semantischen Eigenschaften der Partizipien selbst gemäß den von Croft vorgeschlagenen Parametern der 'Stativität' und der 'Persistenz', so zeigt sich, dass z.B. die Partizipien Präsens von Vorgangs- und Handlungsverben (z.B. fallend in fallende Blätter, beendend in das die Diskussion schließlich beendende Machtwort) weder stativ noch persistent interpretiert werden; sie haben also keine der beiden semantischen Eigenschaften, die für die prototypischen Adjektive charakteristisch sind.

Als infinite Formen haben die attributiven Partizipien der Vergleichssprachen grundsätzlich keine morphologischen Tempus- und Modus-Exponenten; auch der Ausdruck von Aspekt unterliegt, wie z.B. für das Englische festgestellt (vgl. Quirk et al. 1985, S. 1263) starken Beschränkungen. Im Vergleich zu entsprechenden Relativsätzen kann also weder eine zeitliche Situierung (qua Tempusinterpretation) noch eine Modalisierung (qua Modusinterpretation) des Ereignisses, das als referentieller Anker fungiert, vorgenommen werden. (Hier können wir in der Tat von Ereignissen sprechen, nicht nur unspezifisch von Sachverhalten.) Es wird lediglich Überlappung bzw. Vorzeitigkeit zwischen verankerndem Ereignis und Ereignis des einbettenden Satzes angezeigt jeweils durch Partizip

\footnotetext{
${ }^{11}$ Ich gehe nicht auf alle Faktoren in der Abwägung zwischen 'Verbalität' und 'Nominalität' ein, u.a. nicht auf die Frage der Übernahme verbaler Valenzstellen durch das Partizip.

${ }^{12}$ Allerdings ist bei den englischen „,gerund-participials“ (Huddleston/Pullum 2002) der syntaktische Interpretationsspielraum (attributiv? adverbial? gerundial?) oft beträchtlich. Die Durchsicht von Belegen zeigt, dass adnominale being + Adjektiv-Fügungen meist innerhalb von syntaktisch nur locker angebundenen with-Konstruktionen vorkommen wie in diesem Beleg. Generell hat das Englische durchaus auch Züge einer Nominalisierungs-Strategie für propositionale Information, wenn man an die zahlreichen infiniten Konstruktionen denkt.
} 
Präsens bzw. Partizip Perfekt. Dabei können selbstverständlich Temporaladverbialia die Betrachtzeit bzw. die Ereigniszeit genauer beschränken wie etwa in die vor einer Stunde zu Boden gefallenen Blätter; auch periphrastisch ausgedrückte Zeitverhältnisse können gegebenenfalls übernommen werden; dies ist im Deutschen aber stilistisch hoch markiert:

(20) die der damaligen westlichen Industriegesellschaft Widerstand angekündigt habenden Jungen und Studierenden hatten (...) (Die Presse 23.11.1991; vgl. Pakkanen-Kilpiä 2004, S. 205)

(21) Aus dem Rennen ist das an sich für Eisbewerbe gedacht gewesene Kufstein. (Tiroler Tageszeitung 05.07.1997; vgl. Pakkanen-Kilpiä 2004, S. 156)

Deontische Modalisierung kann im Deutschen durch das Gerundivum, also das Partizip Präsens des $z u$-Infinitivs, wie in die später zu zerstörende Stadt ausgedrückt werden allerdings mit stark eingeschränkter Nutzung. Auch das Ungarische verfügt über entsprechende Gerundialformen, die mithilfe des Suffixes -andó/-endö gebildet werden.

Die genannten Unterschiede gegenüber Relativsyntagmen, vor allem die Beschränkung auf Subjektsattribuierung und die fehlende Tempus-/Modus-Repräsentation waren der Grund, warum in Zifonun (2000) die attributiven Partizipialkonstruktionen der Kernkontrastsprachen, die ja alle mit Relativsätzen koexistieren, nicht als Relativinfinite eingestuft wurden. Grundsätzliche Unterschiede im Sprachtyp - hier zwischen 'nominalisierendem' und 'subordinierendem' Typ der Einbettung propositionaler Information - würden verwischt, wenn wir die Relativinfinite etwa des Türkischen und die Partizipialkonstruktionen unserer Vergleichssprachen demselben Konstrukt zuordneten. Die Partizipialkonstruktionen der Vergleichssprachen sind als funktional vergleichbare, aber konstruktionell beschränkte und markierte sekundäre Formen neben den Relativsyntagmen zu bewerten.

Damit sollen aber keineswegs die bestehenden Unterschiede zwischen den attributiven Partizipialkonstruktionen in den Vergleichssprachen nivelliert werden. Dazu nur kurze Hinweise: Im Deutschen (wie im Ungarischen) werden auch durch Komplemente und Supplemente ausgebaute Partizipialphrasen wie komplexe Adjektivphrasen grundsätzlich pränominal gestellt; beide Formen zeigen auch in der Linearisierung der Phrasenteile keinerlei Unterschiede (ein seiner Frau immer treuer Ehemann wie ein seinem Gewissen immer folgender Mensch). Im Englischen (wie im Französischen und überwiegend auch im Polnischen) stehen erweiterte Partizipialphrasen postnominal. Im Englischen ist dies die markiertere der beiden möglichen Stellungen für adjektivische/adnominale Modifikatoren, die einerseits durch die phrasale Komplexität motiviert ist, die andererseits aber deutlich mit der markierteren funktionalen Bestimmung einer Zuschreibung temporärer Eigenschaften bzw. einer referentiellen Verankerung durch ein Ereignis, somit eine temporär gültige Entität, korreliert ist. Dies ergibt sich z.T. ex negativo aus der Tatsache, dass die pränominale Position (vgl. Quirk et al. 1985, S. 1242 ff.; Huddleston/Pullum 2002, S. 445) auf die Zuschreibung permanenter oder zumindest charakteristischer Merkmale eingeschränkt ist und daher bestimmte Modifikatoren (ready, afraid, meist: available), die aufgrund ihrer lexikalischen Bedeutung stark mit temporärem Status assoziiert sind, in pränominaler Position nicht zugelassen sind und andere Modifikatoren (present, involved, concerned) in pränominaler Position eine permanent-charakterisierende, in postnominaler dagegen eine temporäre Lesart haben. Nur die postnominale 
Position lässt sowohl permanente als auch temporäre Modifikation zu, daher stehen die Partizipialphrasen wie die Relativsätze postnominal. Da im Englischen auch in Relativsätzen der Subordinator fehlen kann, besteht neben der linearen in vielen Fällen eine starke strukturelle Analogie zwischen Partizipial- und Relativkonstruktionen; nur das Fehlen des Finitums scheint beide zu unterscheiden; vgl.
(22) a proposal which the President made last year a proposal the President made last year a proposal made by the President last year

\author{
[Relativsatz mit Subordinator] \\ [Relativsatz ohne Subordinator] \\ [Partizipialkonstruktion]
}

Diese hier aus Platzgründen nur auf den Vergleich zwischen dem Deutschen und dem Englischen reduzierte Betrachtung zeigt, dass auch bei den Kernkontrastsprachen die adnominale Partizipialkonstruktion - obschon grundsätzlich nicht als Relativinfinit eingeordnet - variiert zwischen einer vollständigen Konformität mit der Adjektivphrase bei gleichzeitiger Differenz gegenüber dem Relativsatz (wie im Deutschen) und einer starken Übereinstimmung mit dem Relativsatz bei gleichzeitiger Differenz gegenüber dem (unmarkierten, unerweiterten) attributiven Adjektiv (wie im Englischen).

\subsection{Die Korrelation zwischen den Parametern 'Subordination', 'Leerstellenfüllung' und 'Hierarchie syntaktischer Funktionen'}

Im Folgenden werden die drei Parameter bestimmt und sukzessive im Hinblick auf die Vergleichssprachen und insbesondere das Deutsche miteinander in Beziehung gesetzt.

Beim Parameter 'Subordination' geht es darum, wie die syntaktische Beziehung zwischen dem Bezugsausdruck und dem Relativsyntagma an der sprachlichen Oberfläche zum Ausdruck kommt. Dabei wird angenommen, dass nur dann (eindeutig) ein Relativsyntagma vorliegt, wenn das Syntagma als syntaktisch subordiniert zu betrachten ist. Grenzfälle wie etwa die deutschen Verbzweit-,Relativsätze“ werden hier nicht betrachtet (vgl. dazu Blühdorn und Ravetto in diesem Band). Die Parametrisierung im Sprachvergleich betrifft dann die Frage, ob überhaupt ein overter Marker der Subordination des Relativsyntagmas zur Verfügung steht und wenn ja, ob dieser affixal oder lexikalisch realisiert ist. Während es z.B. im Japanischen keinen syntaktischen Ausdruck für die Subordination von Relativsyntagmen gibt, überwiegen in den europäischen Sprachen Relativsyntagmen mit einem in aller Regel lexikalischen Subordinator, affixale Subordination gibt es im Baskischen und bei infinitem Relativsyntagma im Türkischen. Im Englischen und den skandinavischen Sprachen kann in bestimmten Fällen die Setzung des Subordinators unterbleiben, dän./norw./schw. som, engl. that können - nur in restriktiven Relativsätzen - weggelassen werden, wenn nicht über die Subjektstelle relativiert wird. Lexikalische Subordinatoren wiederum kann man im Anschluss an Lehmann in drei Typen einteilen: Relativpartikeln, rein attribuierende Relativpronomina und leerstellenfüllende Relativpronomina. ${ }^{13}$ (Daneben sind die Relativadverbien zu nennen, die speziell zur Relativierung über adverbiale Funktionen dienen wie dt. wo.) Relativpartikeln sind unveränderliche Ausdrücke, die lediglich anzeigen, dass subordiniert wird; insbesondere sind sie keine syntaktischen Repräsentanten der semantischen Leerstelle im Re-

\footnotetext{
${ }^{13}$ Lehmann (1984, S. 97 ff.) spricht von „nicht-resumptiven“ (statt „rein attribuierenden“) und von „,resumptiven“" (statt ,leerstellenfüllenden“) Pronomina.
} 
lativsyntagma. Das engl. that und das festlandskandinavische som sind Beispiele für Relativpartikeln:

(23) the book that the girl read [ $]^{14}$

Rein attribuierende Relativpronomina wie im Arabischen, Swahili und weiteren afrikanischen Sprachen zeigen Kongruenz mit dem Bezugsnomen, verdeutlichen aber nicht die syntaktische Funktion im Relativsatz (vgl. Lehmann 1984, S. 97 ff.).

Viele europäische Sprachen haben leerstellenfüllende Relativpronomina, also veränderliche Ausdrücke, die mit ihrem Bezugswort in Genus und Numerus korrespondieren, somit einerseits auch attribuierend sind, und die andererseits aber z.B. durch ihren Kasus die syntaktische Funktion anzeigen und insofern die semantische Leerstelle im Relativsyntagma „direkt" syntaktisch repräsentieren, und zwar als primäre oder sekundäre Satzglieder. Die einzelnen Formen sind gemäß einem Flexionsparadigma systematisch aufeinander bezogen. Typische Beispiele sind die deutschen (der/die/das) und die polnischen Relativpronomina (który/która/które). Beide Sprachen zeigen zum einen die Abstimmung mit dem Kernsubstantiv bezüglich Genus und Numerus an, zum anderen die Funktion des syntaktischen Repräsentanten der semantischen Leerstelle im Relativsatz durch Kasus, wobei auch Kombinationen aus Präposition + Phrase im regierten Kasus an der relativsatzeinleitenden Position möglich sind; man vergleiche exemplarisch die polnischen Beispiele für die Genusabstimmung in Subjektsfunktion (24), die Numerusabstimmung in der Funktion des indirekten Objekts (25) und ein Beispiel mit einer präpositionalen Füllung (26):

(24) mężczyzna / kobieta / dziecko, który / która / które dużo czyta 'der Mann / die Frau / das Kind, der / die / das viel liest'

(25) mężczyźni, którym wierzysz

'die Männer, denen du glaubst'

(26) książka, o której mówiłaś

'das Buch, über das du sprachst'

Auch die durch Präfigierung von $a$ - aus den Interrogativa abgeleiteten Relativa des Ungarischen sind leerstellenfüllend, allerdings entfällt Genuskorrespondenz. Stattdessen spielen andere referenzbezogene Eigenschaften des Antezedens (Kernsubstantivs) eine Rolle, nämlich die Kategorien [ \pm personal], $[ \pm$ individuativ] sowie $[ \pm$ spezifisch] (vgl. Kenesei et al. 1998, S. 40), die Unterscheidungen in der sprachübergreifend gültigen erweiterten Belebtheits- bzw. der Definitheitshierarchie erfassen (vgl. Croft 2003, S. 128 ff., Zifonun 2007). Bei personenbezeichnendem Antezedens steht aki (27), bei Stoffbezeichnungen [-individuativ] oder unspezifisch referierendem Antezedens steht ami 'das / was' (28), Individuativa bzw. spezifisch referierende Nominale werden mit amely 'welcher', umgangssprachlich auch mit ami, relativiert (29).

\footnotetext{
${ }^{14}$ In Grammatiken des Englischen wird häufig auch relatives that als Relativpronomen eingeordnet (vgl. Quirk et al. 1985, S. 366). In neueren Ansätzen wird unter dem Einfluss sprachvergleichender Untersuchungen der Unterschied zwischen den pronominalen wh-Formen und that hervorgehoben. So heißt es bei Huddleston/Pullum (2002, S.1037) beispielsweise ,that marks the clause as subordinate, the subject position [über die im entsprechenden Beispiel relativiert wird, G.Z.] is empty.“
} 
(27) az a lány, aki a könyvet olvassa

'jenes Mädchen, das das Buch liest'

(28) a pénz, amit kölcsönöztél

'das Geld, das du liehst'

(29) az a könyv, amit / amelyet Anna olvasott

'jenes Buch, das Anna las'

Im Prinzip ähnlich verhalten sich die ebenfalls interrogativbasierten englischen Relativpronomina who/which: Es unterbleibt ebenfalls Genus- und darüber hinaus auch Numerusabstimmung zugunsten einer Unterscheidung nach der Kategorie [ \pm personal] des Antezedens.

(30) the girl / the girls who read the book

(31) the book which the girl read

Nur who ist veränderlich und zeigt die Differenzierung in Subjektivus (who), Possessivus (whose) und Objektivus (whom) an; in bestimmten Varietäten allerdings finden wir nur (noch) die Opposition zwischen „common case“ (who) und Possessivus. which zeigt keine Kasusdifferenzierung - für den Possessivus muss suppletiv whose oder die analytische Form of which stehen; dennoch unterscheidet es sich von der Partikel that zum einen durch die spezifische Attribuierung nur an nicht-belebte Antezedentien, zum anderen dadurch, dass es wie whom mit einer Präposition zu einer regulären den Relativsatz einleitenden PP verbunden werden kann, während bei that nur „Präpositionsstranden“ möglich ist:

(32) statements about which readers can agree / which readers can agree about

(32a) statements *about that readers can agree / that readers can agree about

Wie im Englischen ist in den romanischen Sprachen der Übergang von veränderlichen Relativpronomina zu unveränderlichen Relativpartikeln zu beobachten, mit dem Unterschied, dass im Englischen zwei getrennte Bestände (die wh-Pronomina mit der Tendenz zum Kasusabbau und die Partikel that) vorliegen, während im Französischen, Italienischen und den iberoromanischen Sprachen die einfachen Formen ${ }^{15}$ des einen auf die lat. $q u$-Interrogativa/Relativa zurückgehenden Inventars zunehmend nicht mehr als ein Paradigma begriffen werden, sondern als jeweils isolierte Formen mit einem jeweiligen beschränkten Anwendungsbereich. Bei den morphologisch einfachen Relativa des Französischen gibt es eine auffällige Korrelation zwischen syntaktischer Funktion und der Wahl des Subordinators: Die Subjektsfunktion wird (ohne Genus- / Numeruskorrespondenz und ohne Beachtung des Belebtheitskontrastes) von qui wahrgenommen (33), die des direkten Objekts und des Prädikativkomplements von que $(34,35)$, als Komplement einer Präposition erscheint qui ([+belebt]) bzw. quoi ([-belebt]) (36). Statt der Verbindung de + qui / quoi - aber auch genereller zur Relativierung von Präpositionalphrasen steht dont (37). qui und quoi erscheinen somit, vor allem aufgrund ihrer Kombinierbarkeit mit Präpositionen, am ehesten noch als Relativpronomina, que hingegen, das wie

\footnotetext{
${ }^{15}$ Im Französischen, Italienischen, Spanischen, Portugiesischen stehen jeweils daneben komplexe (aus Artikel + Interrogativum zusammengesetzte) Formen zur Verfügung wie frz. lequel, ital. il quale, span. el quelel cual, port. o qual; diese bleiben hier außer Betracht.
} 
englisch that mit dem Komplementierer übereinstimmt und nicht als Komplement einer Präposition stehen kann, als Relativpartikel; anders als englisch that ist die 'Relativpartikel' que jedoch standardsprachlich nicht für alle syntaktischen Funktionen auf Satzebene verallgemeinert. dont kann als relativiertes Gegenstück zu en und damit als Relativadverb gelten: ${ }^{16}$

(33) mon père / ma mère / mes parents qui venai(en)t de Paris 'mein(e) Vater / Mutter / Eltern, der / die aus Paris kam(en)'

(34) le pays / les hommes que je connais depuis longtemps 'das Land / die Leute, das / die ich seit langem kenne'

(35) l'homme / la femme que Pierre / Marie était 'der Mann / die Frau, der / die Pierre / Marie war'

(36) l'homme / la chose à qui / à quoi il n'avait pas pensé 'der Mensch / die Sache, an den / an die er nicht gedacht hatte'

(37) l'homme / le café dont je connais l'adresse 'der Mann / das Café, dessen Adresse ich kenne'

Im Italienischen ist die Grammatikalisierung der ursprünglichen Objektivus-Form che zur Relativpartikel weiter fortgeschritten als im Französischen: che wird als Subjekt und direktes Objekt gebraucht, cui primär als Komplement von Präpositionen, daneben als indirektes Objekt und Possessivus. ${ }^{17}$

Gehen wir nun über zur Frage der Leerstellenfüllung: Die syntaktische Leerstelle, die der semantischen Leerstelle bei Relativierung entspricht, kann im Prinzip leer bleiben oder gefüllt werden. Beim ersten Fall, der 'Lückenbildung', hat x, die semantische Leerstelle, keinen Vertreter im Relativsyntagma: es fehlt also der pronominale Anschluss an das Antezedens. Ein solcher pronominaler Anschluss kann entweder ein „relativer“" (Relativpronomina) sein oder ein „resumptiver“ (Personal- oder Demonstrativpronomina bzw. deiktische Adverbien wie dt. $d a$ ). Die Relativpronomina des Deutschen, Polnischen und Ungarischen sind voraussetzungsgemäß und obligatorisch leerstellenfüllend, sie kookkurrieren nicht mit einem ,anderen“ syntaktischen Repräsentanten der semantischen Leerstelle, also einem Resumptivum, wie das ungrammatische deutsche Beispiel (38) verdeutlicht:

(38) *der Mann, den ich ihn sehe / dem ich ihm das Buch gegeben habe

Resumptiva können auftreten, wenn eine Relativpartikel als Subordinator fungiert. Möglich wäre ihre Setzung auch, wenn kein Subordinator vorhanden ist; dies ist aber in den europäischen Sprachen nicht belegt und insgesamt wohl sehr selten. ${ }^{18}$ Das Vorhandensein einer Relativpartikel führt aber, wie das Phänomen der Lückenbildung eben zeigt,

\footnotetext{
${ }^{16}$ Es kann also durchaus auch die in den Grammatiken des Französischen übliche Meinung aufrecht erhalten werden, es liege ein suppletives Paradigma von Relativpronomina vor (Subjektivus: qui, Possessivus: dont, Komplement einer Präposition qui/quoi).

${ }^{17}$ In Schwarze (1995, S. 452 ff.) wird die Problematik der Einordnung der italienischen Relativierer, insbesondere von che, ausführlich erörtert.

${ }^{18}$ Ein konstruiertes Beispiel im Englischen wäre: the man [] I saw him. Nach de Vries (2002, S. 165) gilt allgemein: „Resumptive pronouns almost always occur in addition to a relative particle or a relative marker.“"Er nennt nur vier Ausnahmen aus einem Sample von 172 Sprachen.
} 
nicht grundsätzlich zur Setzung eines resumptiven Pronomens. Im Englischen und den skandinavischen Sprachen, erscheinen bei Relativsätzen, die durch Relativpartikeln subordiniert werden, keine Resumptiva. Wir kommen auf zusätzliche Bedingungen für die Setzung von Resumptiva zurück. Die Korrelation zwischen den Alternativen 'Lückenbildung' versus 'Pronominalisierung' auf der einen Seite und der Wahl des Subordinators halten wir in Tabelle 1 fest:

\begin{tabular}{|c|c|c|}
\hline Subordinator & Pronominalisierung & Lückenbildung \\
\hline ohne overten Subordinator & 19 & $\begin{array}{l}\text { Englisch, skandinavische } \\
\text { Sprachen } \\
\text { engl.: the man [] I saw [] }\end{array}$ \\
\hline Relativpronomen & $\begin{array}{l}\text { „,relativ“ } \\
\text { Deutsch, (Französisch), Pol- } \\
\text { nisch, Ungarisch } \\
\text { dt. der Mann, den ich sah }\end{array}$ & \\
\hline Relativpartikel & $\begin{array}{l}\text { „resumptiv“ } \\
\text { Substandard zahlreicher euro- } \\
\text { päischer Sprachen } \\
\text { frz. l'homme que je le vois }\end{array}$ & $\begin{array}{l}\text { Englisch, skandinavische } \\
\text { Sprachen } \\
\text { engl.: the man that I saw [] }\end{array}$ \\
\hline
\end{tabular}

Tabelle1: Die Korrelation zwischen Subordinator und Leerstellenfüllung

Standardsprachlich kommt die Kookkurrenz des Subordinators mit einem Resumptivum z.B. im Griechischen vor; hier ist der Relativsatzeinleiter die Partikel pu (vgl. Lehmann 1984, S. 88 ff.). Ansonsten finden wir sie vor allem in dialektalen oder substandardsprachlichen, gesprochenen Varietäten europäischer Sprachen. Das gemeinsame Vorkommen von Subordinator und Resumptivum kann gemäß der in Tabelle 1 festgehaltenen Korrelation auch als eine Art Test dafür dienen, ob der Subordinator als Pronomen oder als Partikel einzuschätzen ist. In den beiden soeben erörterten romanischen Sprachen, Französisch und Italienisch, wird die Hypothese, que/che sei - bezogen auf das Diasystem - auf dem Weg zur Relativpartikel durch substandardsprachliche Befunde wie die beiden folgenden bestätigt:

(38a) l'homme que je le vois / que je lui ai donné le livre 'der Mann, den ich sehe / dem ich das Buch gegeben habe'

(39) (...) uno di quelli che gli si legge in faccia l'arte di far carriera (...) 'einer von denen, dem die Kunst des Karrieremachens ins Gesicht geschrieben ist' (vgl. Schwarze 1995, S. 452)

An dieser Stelle nun muss als Faktor für die Setzung eines Resumptivums auch als dritter Parameter die Hierarchie syntaktischer Funktionen ins Spiel gebracht werden. Diese

\footnotetext{
${ }^{19}$ Die Zelle 'ohne overten Subordinator/Pronominalisierung' ist nicht belegt, aber grundsätzlich belegbar, vgl. Fußnote 18. Die graue Unterlegung der Zelle 'Relativpronomen/Lückenbildung' dagegen deutet darauf hin, dass diese Werte miteinander absolut inkompatibel sind.
} 
geht auf die einflussreiche Untersuchung von Keenan/Comrie (1977) zurück und lautet in der ursprünglichen Version wie folgt:

(i) Zugänglichkeitshierarchie (Accessibility Hierarchy):

$\mathrm{SU}>\mathrm{DO}>\mathrm{IO}>\mathrm{OBL}>\mathrm{GEN}>\mathrm{OCOMP}($ Keenan/Comrie 1977, S. 66)

mit folgender Legende: ,> “ steht für 'ist für Relativierung zugänglicher als', SU für 'Subjekt', DO für 'direktes Objekt', IO für 'indirektes Objekt', OBL für 'Obliquus', GEN für

'Genitiv' bzw. 'Possessivus' und OCOMP für 'Vergleichsobjekt'/”object of comparison"'

Die Hierarchie, die auf der Auswertung eines Samples von etwa 50 Sprachen beruhte, zielte primär darauf $\mathrm{ab}$, die sprachübergreifenden Ordnungsprinzipien für die Relativierbarkeit von Teilen des Relativsyntagmas unter Angabe der jeweiligen syntaktischen Funktionen zu formulieren. Dabei gilt folgende Beschränkung (vgl. Zifonun 2001, S. 41):

(ii) Die Menge der in einer Sprache (mittels einer bestimmten Relativstrategie) relativierbaren syntaktischen Funktionen bildet jeweils ein kohärentes Segment aus der einschlägigen Hierarchie - beginnend mit der hierarchiehöchsten Funktion: Wenn eine Funktion $F_{i}$ relativierbar ist, so sind alle in der Hierarchie der Funktion $F_{i}$ vorgeordneten Funktionen ebenfalls relativierbar.

In all unseren Vergleichssprachen kann bei fast allen Positionen der Hierarchie Relativierung erfolgen: Nur beim letzten Element, den Vergleichskonstruktionen (Funktion OCOMP) verhalten sich die Sprachen unterschiedlich (vgl. dazu und zu Lehmanns Version der Zugänglichkeitshierarchie mit der Unterscheidung zwischen Satzgliedfunktionen und adnominalen Funktionen Zifonun 2001, S. 41 ff.).

Für unseren Zusammenhang ist aber ein Korollar zu der Hierarchie (i) von besonderem Interesse, nämlich der Zusammenhang mit Pronominalisierung versus Lückenbildung: Wie die Befunde aus vielen Sprachen zeigen, ist die Wahrscheinlichkeit für das Auftreten eines Resumptivums umgekehrt proportional zur Hierarchieposition der relativierten Stelle. In Zifonun (2001, S. 47 ff.) wird für die Kontrastsprachen und weitere europäische Sprachen gezeigt, dass (standardsprachlich oder im Substandard) häufig ab der Position IO die Setzung eines resumptiven Pronomens obligatorisch oder favorisiert ist, während bei Relativierung von SU grundsätzlich, bei der von DO häufig Lückenbildung möglich ist.

Auf vergleichbare Effekte im Deutschen wurde in Zifonun (2001) nicht eingegangen, und zwar unter der Prämisse, dass das Standarddeutsche mit seinen leerstellenfüllenden Relativpronomina überhaupt keinen Bedarf und keine Möglichkeit für die Setzung von Resumptiva hat. Dies ist zutreffend. Allerdings gilt auch für das Deutsche wie für andere europäische Sprachen, dass die flektierenden leerstellenfüllenden Relativa, die als eines ihrer bemerkenswerten arealen Spezifika, als ein Merkmal des Standard Average European, vgl. Haspelmath (2001, S. 1494 f.), betrachtet werden, in erster Linie ein Phänomen des geschriebenen Standards sind und daher möglicherweise einen durch normierende Einflüsse konservierten Sprachstand reflektieren, oder gar einen, der ,nicht natürlich“ ist (Fleischer 2004b, S. 81). Der sprachgeschichtliche Befund (vgl. Zifonun 2003) und neuere Untersuchungen zur Relativsatzgrammatik in deutschen Dialekten (Fleischer 2004a, b) bestätigen, dass auch hier Relativsätze eher nicht bzw. nicht nur durch flektierte Einheiten eingeleitet werden, und dass auch hier ab der Position OBL in der Regel, in 
manchen Varietäten auch ab IO ein leerstellenfüllendes Element (ein ,relatives“ oder „resumptives“ Pronomen) hinzutritt.

Bekanntlich ist auch in der standardnäheren Umgangssprache (in ihren gesprochenen oder etwa in E-Mails, Chats verbreiteten Formen) wo als Partikel generalisiert. Setzung von wo ohne Leerstellenfüllung scheint problemlos, wenn auch normwidrig, für die beiden obersten Positionen von Hierarchie (i), also SU und DO, möglich zu sein.

(40) „Eddie the Eagle - der Mann, wo schneller als der Bus war“ (http://www.steppenhahn.de/elt/2001.html)

(41) (...) wird aber später von Clarks Feinden den 2 (die Frau und der Mann wo Clark in die Phantomzone befördert hat) getötet.

(www.fan-forum.de/smf/index.php/topic,6002.0.html)

Andererseits ist häufig für $\mathrm{OBL}$ (also präpositional realisierte Komplemente/Supplemente) die Aufspaltung in die Partikel wo $+d a$-Präpositionaladverb zu beobachten, oder auch, mit regionaler Zuordnung zum Norden bzw. Nordwesten, wo + ,gestrandete“ Präposition statt dem aus wo und Präposition zusammengesetzten Präpositionaladverb:

(42) Also das ist ja ne Sache, wo ich generell dagegen bin. (Internetbeleg, vgl. DudenGrammatik 2005, S. 1043)

(43) Also wenns eine Bettgeschichte werden soll, wo ich generell gegen bin, sollte man erst gar nicht flirten und sich die Vorarbeit machen (...). (http://www.hilfe-forum.eu/sitemap/ t-40010.html, 12.05.2004)

Stärker markiert ist die Kombination von flektierendem der/die/das $+w o$; man vergleiche auch hier zunächst mit (44) einen Beleg mit präpositionaler Rektion. Die Leerstellenfüllung erfolgt hier ,relativ“ (im Sinne von Tabelle 1); dennoch ist eine Partikel gesetzt, zu einer „resumptiven“ Form vgl. dagegen Beleg (45) aus dem Basler Dialekt.

(44) ist dit der Daniel mit dem wo ich die ganze Zeit gemailt habe? (http://www.gruenderszene.de/?feed $=\mathrm{rss} 2 \& \mathrm{p}=86$ )

(45) die Lyt wo mer iber sie gschwätzt händ 'die Leute, wo wir über sie geschwätzt haben' (vgl. Fleischer 2004b, S. 76)

Bei den beiden obersten Rängen der Hierarchie kann auf Leerstellenfüllung verzichtet werden, in der Obliquusposition wird durch die Setzung der Präposition die Position der relativierten Stelle hinreichend verdeutlicht, unabhängig davon, ob die Leerstelle gefüllt ist wie in (42), (44), (45) oder nicht wie in (43), sofern hier wo als Partikel interpretiert wird. Was aber ist mit IO? Die Relativierung dieser Position ist wie in den Dialekten auch im Substandard problematisch. Auch bei Relativierung von IO wird nach meiner Einschätzung, die durch empirische Untersuchungen weiter abzusichern ist, in informellem Register tendenziell die Leerstelle besetzt.

(46) der Mann, ?(dem) wo ich ziemlich viel Geld schulde

Die Besonderheit des IO besteht im Deutschen darin, dass es wie die beiden höheren Positionen durch Kasus kodiert wird, aber sowohl morphologisch als auch in der syntaktischen Funktionalität von den Komplementen mit den „direkten Kasus“ Nominativ und Akkusativ (vgl. z.B. Eisenberg 2004a, S. 172) deutlich abgegrenzt ist. Im Englischen dagegen ist das indirekte Objekt entweder bei Realisierung durch „common case“ nur 
konfigurationell-linear vom DO unterschieden (wie in I gave the man the book) oder es ist mit der Präposition to, formal als Obliquus realisiert (I gave the book to the man). Bei Relativierung von IO unter Lückenbildung ist das explizitere Verfahren mit Präposition deutlich bevorzugt:

(47) The person (that) I sent the book ?(to) has not acknowledged receiving it. (vgl. Quirk et al. 1985, S. 726)

Möglicherweise ist auf diesem Hintergrund der Kontrast von Deutsch und Englisch (bzw. den skandinavischen Sprachen) zu erklären. Dort führt die Setzung von Relativpartikeln (oder auch das Fehlen des Subordinators) in keiner der satzbezogenen Positionen SU, DO, IO, OBL zur Füllung der Leerstelle, nur die adnominale GEN-Position ist schlecht mit Lückenbildung vereinbar. ${ }^{20}$ Wie oben ausgeführt, ist bei SU und DO sprachübergreifend kein Resumptivum gefordert und bei OBL kann die Präposition „ausreichen“. Da im Englischen das indirekte Objekt unter Relativierung in der Regel als Obliquus erscheint, besteht insgesamt kein Bedarf für resumptive Leerstellenfüllung.

\section{Schlussbemerkung}

In diesem Beitrag wurde versucht, am Beispiel des Relativsatzes Grundkonzepte des Projekts „Grammatik des Deutschen im europäischen Vergleich“ möglichst explizit und bezogen auf die grammatischen Fakten der Vergleichssprachen zu erläutern. Eher unter der Hand ist dabei eine Art Fallstudie zur Integration von Sprachtypologie, Sprachvergleich und einzelsprachlicher Grammatikschreibung entstanden, wie sie in unserem Projekt angestrebt wird. Es sollte deutlich geworden sein, dass die aus der Sprachtypologie abzuleitenden Parametersetzungen der Ergänzung durch die detaillierte einzelsprachliche Phänomenbeschreibung bedürfen. So verstehen wir konkurrierende Formen wie etwa Partizipialkonstruktion und Relativsatz besser, wenn wir beide vor dem Hintergrund der funktionalen Domäne der 'referentiellen Modifikation durch einen verankernden Sachverhalt' und der Option nicht-finiter und finiter Realisierung betrachten. Aber erst die Gegenüberstellung der grammatischen Eigenschaften, die die Konstruktionstypen etwa im System des Deutschen oder Englischen haben, verschafft Klarheit über ihren jeweiligen Stellenwert und das Verhältnis zwischen ihnen. Ebenso wird die Wahl zwischen den Alternativen Pronominalisierung oder Lückenbildung im Relativsatz, die wir als solche im typologischen Kontext erst deutlich erkennen mögen, erst durch die Verknüpfung einer ganzen Reihe von Faktoren der einzelsprachlichen Grammatik erklärbar sein.

\section{Literatur}

Croft, William (1991): Syntactic Categories and Grammatical Relations: The Cognitive Organization of Information. Chicago: University of Chicago Press.

Croft, William (2003): Typology and Universals. $2^{\text {nd }}$ ed. Cambridge: Cambridge University.

Dixon, Robert. M. W. (1977): Where have all the adjectives gone? In: Studies in Language 1, S. 19-80.

\footnotetext{
${ }^{20}$ Auch im Schwedischen ist außer bei Verben wie give, promise bei Relativierung des IO ohne Subordinator oder mit der Partikel som in der Regel präpositionale Realisierung gefordert (Smits 1989, S. 426); im Norwegischen ist die nicht-präpositionale Variante möglich, ,,although some speakers strongly prefer the prepositional variant"“ (Smits 1989, S. 390).
} 
Eisenberg, Peter (2004a): Grundriß der deutschen Grammatik. Das Wort. 2. Aufl. Stuttgart/ Weimar: Metzler.

Eisenberg, Peter (2004b): Grundriß der deutschen Grammatik. Der Satz. 2. Aufl. Stuttgart/ Weimar: Metzler.

Fleischer, Jürg (2004a): A Typology of Relative Clauses in German Dialects. In: Kortmann, Bernd (Hg.): Dialectology Meets Typology: Dialect Grammar from a Cross-Linguistic Perspective. Berlin: Mouton de Gruyter. S. 211-243. (= Trends in Linguistics: Studies and Monographs 153).

Fleischer, Jürg (2004b): Zur Typologie des Relativsatzes in den Dialekten des Deutschen. In: Patocka, Franz/Wiesinger, Peter (Hg.): Morphologie und Syntax deutscher Dialekte und Historische Dialektologie des Deutschen: Beiträge zum 1. Kongress der Internationalen Gesellschaft für Dialektologie des Deutschen, Marburg/Lahn, 5.-8. März 2003. Wien: Edition Praesens. S. 60-83.

Fox, Barbara A./Thompson, Sandra A. (1990): A discourse explanation of the grammar of relative clauses in English conversation. In: Language 66/2, S. 297-316.

Frajzyngier, Zygmunt (1999): Domains of Point of View and Coreferentiality: System Interaction Approach to the Study of Reflexives. In: Frajzyngier, Zygmunt/Curl, Traci S. (eds.): Reflexives. Forms and Functions. Amsterdam/Philadelphia: Benjamins. S. 125-153. (= Typological Studies in Language 40).

Givón, Talmy (1981): Typology and Functional Domains. In: Studies in Language 5, S. 163-193.

Gunkel, Lutz/Zifonun, Gisela (i.E.): Grammatik des Deutschen im europäischen Vergleich: Die Nominalphrase.

Haspelmath, Martin (2001): The European Linguistic Area. Standard Average European. In: Haspelmath, Martin et al. (Hg.): Language Typology and Language Universals. Berlin: de Gruyter. S. 1492-1510. (= Handbücher zur Sprach- und Kommunikationswissenschaft 20/2).

Huddleston, Rodney/Pullum, Geoffrey K. (2002): The Cambridge Grammar of the English Language. Cambridge: Cambridge University Press.

Keenan, Edward L./Comrie, Bernard (1977): Noun Phrase Accessibility and Universal Grammar. In: Linguistic Inquiry 8, S. 63-99.

Kenesei, István/Vago, Robert M./Fenyvesi, Anna/Comrie, Bernard (1998): Hungarian. London/New York: Routledge.

Koptjevskaja-Tamm, Maria (2002): Adnominal Possession in the European Languages: Form and Function. In: Sprachtypologie und Universalienforschung 55, S. 141-172.

Koptjevskaja-Tamm, Maria (2003): Possessive Noun Phrases in the Languages of Europe. In: Plank, Frans (Hg.): The Noun Phrase Structure in the Languages of Europe. Berlin/New York: de Gruyter. S. 621722. (= Eurotyp 20-7).

Kornfilt, Jaklin (1997): Turkish. London/New York: Routledge.

Kratzer, Angelika (1989): Stage Level and Individual Level Predicates. In: Bach, Emmon et al. (eds.): Papers on Quantification. NSF report. University of Massachusetts. Amherst. S. 225-264.

Lehmann, Christian (1984): Der Relativsatz. Typologie seiner Strukturen, Theorie seiner Funktionen, Kompendium seiner Grammatik. Tübingen: Gunter Narr Verlag. (= Language Universals Series 3).

Lehmann, Christian (2004): Sprachtheorie. Erfurt: Universität Erfurt (http://www.uni-erfurt.de/sprachwissenschaft/personal/lehmann/CL_Lehr/Course_materials.html).

Lerner, Jean-Yves/Zimmermann, Thomas E. (2001): Eigennamen. In: Stechow, Arnim von/Wunderlich, Dieter (Hg.) (1991): Semantik: Ein internationales Handbuch der zeitgenössischen Forschung. Berlin: de Gruyter. S. 349-370. (= Handbücher zur Sprach- und Kommunikationswissenschaft 6).

Lyons, John (1983): Semantik. Band 2. München: Beck. 
Motsch, Wolfgang (1966): Syntax des deutschen Adjektivs. 3. Aufl. Berlin: Akademieverlag (= Studia Grammatica 3).

Pakkanen-Kilpiä, Kirsi (2004): Zur Verwendbarkeit des Partizips II als Attribut. Frankfurt etc.: Peter Lang. (= Finnische Beiträge zur Germanistik Bd. 11.)

Prince, Ellen. F. (1981): Toward a Taxonomy of Given-New Information. In: Cole, Peter (Hg.): Radical Pragmatics. New York etc.: Academic Press. S. 223-255.

Quirk, Randolph/Greenbaum, Sidney/Leech, Geoffrey/Svartvik, Jan (Hg.) (1985): A Comprehensive Grammar of the English Language. London: Longman.

Rijkhoff, Jan (2004): The Noun Phrase. Oxford: University Press.

Rijkhoff, Jan (2006): Handout zum Vortrag „Form and Function of Noun Modifiers“. Workshop 'Syntax der Nominalphrase', 16.-17. November 2006. IDS Mannheim.

Schwarze, Christoph (1995): Grammatik der italienischen Sprache. Tübingen: Niemeyer.

Searle, John (1969): Speech Acts. An Essay in the Philosophy of Language. Cambridge: University Press.

Seiler, Hansjakob (1985): Kategorien als fokale Instanzen von Kontinua: gezeigt am Beispiel der nominalen Determination. In: Schlerath, Bernfried (Hg.): Grammatische Kategorien. Funktion und Geschichte. Wiesbaden: Reichert. S. 435-448.

Seiler, Hansjakob (2000): Language Universals Research. Tübingen: Narr. (= Language Universals Series 8).

Smits, Reinier J. C. (1989): Eurogrammar. The Relative and Cleft Constructions of the Germanic and Romance Languages. Dordrecht: Foris Publications.

Taylor, John R. (1996): Possessives in English: An Exploration in Cognitive Grammar. Oxford: Clarendon Press.

de Vries, Marc (2002): The Syntax of Relativization. (pdf) (http://www.let.uu.nl/LOT/)

Wiese, Bernd (2004): Zur Systematisierung der Schwankungen zwischen starker und schwacher Adjektivflexion nach Pronominaladjektiven. Ms., IDS Mannheim. (pdf, 24 pp.)

Zifonun, Gisela (2001): Grammatik des Deutschen im europäischen Vergleich: Der Relativsatz. Mannheim: IDS. (= amades - Arbeitspapiere und Materialien zur deutschen Sprache 3).

Zifonun, Gisela (2001a): Grammatik des Deutschen im europäischen Vergleich. In: Studia Linguistica, Breslau. Acta Universitatis Wratislaviensis XX/2001, S. 171-186.

Zifonun, Gisela (2003): Sprachtypologische Fragestellungen in der gegenwartsbezogenen und der historischen Grammatik des Deutschen, am Beispiel des Relativsatzes. In: Lobenstein-Reichmann, Anja/Reichmann, Oskar (Hg.): Neue historische Grammatiken. Zum Stand der Grammatikschreibung historischer Sprachstufen des Deutschen und anderer Sprachen. Tübingen: Niemeyer. S. 59-85. (= Reihe Germanistische Linguistik 243).

Zifonun, Gisela (2007): „Grammatik des Deutschen im europäischen Vergleich“: 'Belebtheit' als Varianzparameter. Erscheint in: Dębski, Antoni/Fries, Norbert (Hg.): Deutsche Grammatik im europäischen Dialog. Beiträge zum Kongress Krakau 2006. Kraków/Berlin. Online-Publikation: krakau2006.anaman.de.

Prof. Dr. Gisela Zifonun

Institut für Deutsche Sprache

Postfach101621

D-68016 Mannheim

e-mail: zifonun@ids-mannheim.de 